\title{
Quand les contextes se comparent et se parlent
}

\author{
Thomas FORISSIER ${ }^{1}$, Jacqueline BOURDEAU ${ }^{2}$ et Valéry PSYCHÉ \\ ${ }^{1}$ CRREF (EA 4538) - Université des Antilles \\ ${ }^{2}$ Université TÉLUQ
}

\section{Résumé :}

L'idée de départ du projet TEEC consiste à intégrer le contexte dans l'apprentissage des sciences et d'étudier les modalités de cette intégration. Face à un enseignement trop souvent détaché du contexte, le rôle de ce dernier pourrait être mis en lumière au bénéfice des élèves en provoquant un choc entre deux contextes contrastés, dans le cadre de l'étude d'une même notion scientifique. Ce projet s'appuie sur des apprentissages collaboratifs synchrones et asynchrones menés par des élèves de Guadeloupe et du Québec. La méthodologie retenue est de type Design-Based Research (DBR), et se matérialise par la mise en œuvre de plusieurs itérations in situ. Ces itérations portent sur l'histoire sociale, la géothermie, l'éducation au développement durable, et le conte. L'enjeu de ce projet de recherche est d'étudier les relations entre modélisation des contextes externe et émergence des effets de contextes.

\section{Mots-clés :}

Apprentissage contextuel, conception pédagogique, apprentissage collaboratif à distance, recherche basée sur le design, modélisation de contexte, analyse de traces, détection d'émotions, système de tutorat intelligent contextuel.

\begin{abstract}
:
The original idea of the TEEC project is to integrate the context in science learning and to study the modalities of this integration. Faced with an education too often detached from the context, the role of the latter could be highlighted for the benefit of students by causing a clash between two contrasting contexts, within the framework of the study of the same scientific notion. This project is based on synchronous and asynchronous collaborative learning led by students from Guadeloupe and Quebec. The chosen methodology is of the Design-Based Research (DBR) type, and is materialized by the implementation of several in situ iterations. These iterations focus on social history, geothermal energy, education for sustainable development, and storytelling. The challenge of this research is to study the relationships between modeling external contexts and the emergence of context effects.
\end{abstract}

\section{Keywords :}

Context based Learning, instructional design, collaborative distance learning, DesignBased Research, context modeling, trace analysis, detection of emotions, context-aware intelligent tutoring system. 


\section{Introduction}

Cet article décrit un programme de recherche intitulé Technologie Éducative et Enseignement en Contexte (TEEC), qui vise à intégrer le contexte dans les apprentissages et à étudier les effets de cette intégration. Ce programme met en lumière le rôle du contexte pour le bénéfice des élèves en provoquant un choc entre deux contextes contrastés, dans l'étude d'une même notion scientifique. La description du programme contient sa genèse et la problématique, les objectifs et les hypothèses, sa dimension d'apprentissage collaboratif à distance, ainsi que la gestion du projet. La méthodologie retenue est de type Design-Based Research (DBR), et se traduit dans le carde de ce projet par la mise en œuvre de plusieurs itérations in situ, la modélisation du contexte, le développement du calculateur MazCalc, la scénarisation pédagogique, l'analyse épistémique des traces et la détection automatique d'émotions. Ces travaux devraient déboucher sur le design d'un système tutoriel intelligent conscient du contexte (CAITS).

L'article est structuré de la façon suivante : dans un premier temps, les définitions retenues des notions de contexte, de contextualisation didactique, et d'effet de contexte seront présentées. Ensuite la problématique du programme de recherche, ainsi que le plan de mise en œuvre au sein d'un apprentissage collaboratif à distance seront abordés.

\section{Contextes et apprentissages}

\subsection{Contexte, contextualisation didactique et effet de contexte}

En 2013, Delcroix, Forissier et Anciaux présentent un cadre d'analyse opérationnel des phénomènes de contextualisation. Ces auteurs présentent la contextualisation didactique comme un système constitué de pratiques d'enseignement, à l'instar de Blanchet (2009), de processus d'apprentissage et de modélisation des systèmes éducatifs. L'idée que la contextualisation puisse être modélisée par une compréhension fine de ces trois dimensions ainsi que de leurs interactions sera reprise notamment dans les travaux de De Lacaze (2015) et de Odacre et Delcroix (2014). Dans ce cadre, la notion d'effet de contexte est intégrée (Merlo-Leurette et Forissier, 2009) comme un écart de contextes internes entre deux acteurs d'une situation produisant un incident dans les apprentissages. Nous reprenons la definition d'un incident technique critique proposée par Flanagran (1954) : «By an incident is meant any observable human activity that is sufficiently complete in itself to permit inferences and predictions to be made about the person performing the act. »

La différenciation entre les contextes interne et externe au sens de Van Wissen (2013) est structurante, de nombreux travaux s'étant intéressés aux relations multiples entre les contextes et les apprentissages (cf. Figure 1). Certains ont étudié le rôle des contextes externes dans le processus d'apprentissage montrant, par exemple, l'impact des dimensions sociologiques (Duru-Bellat, 2002) ou du genre (Weinburgh, 1995) sur les choix et réussites scolaires. Sauvage Luntadi et Tupin (2012), en s'intéressant aux différents contextes éducatifs représentés comme entourant la situation d'enseignement, s'inscrivent également dans l'étude des relations entre un objet didactique et de ce type de contextes. Les travaux de didactique des sciences s'inscrivant dans les approches authentiques (Schwartz et Crawford, 2006) et basés sur les contextes (King et Ritchie, 2012; Podschuweit et Bernholt, 2017) sont également centrés sur des contextes externes, écologiques et géographiques. 

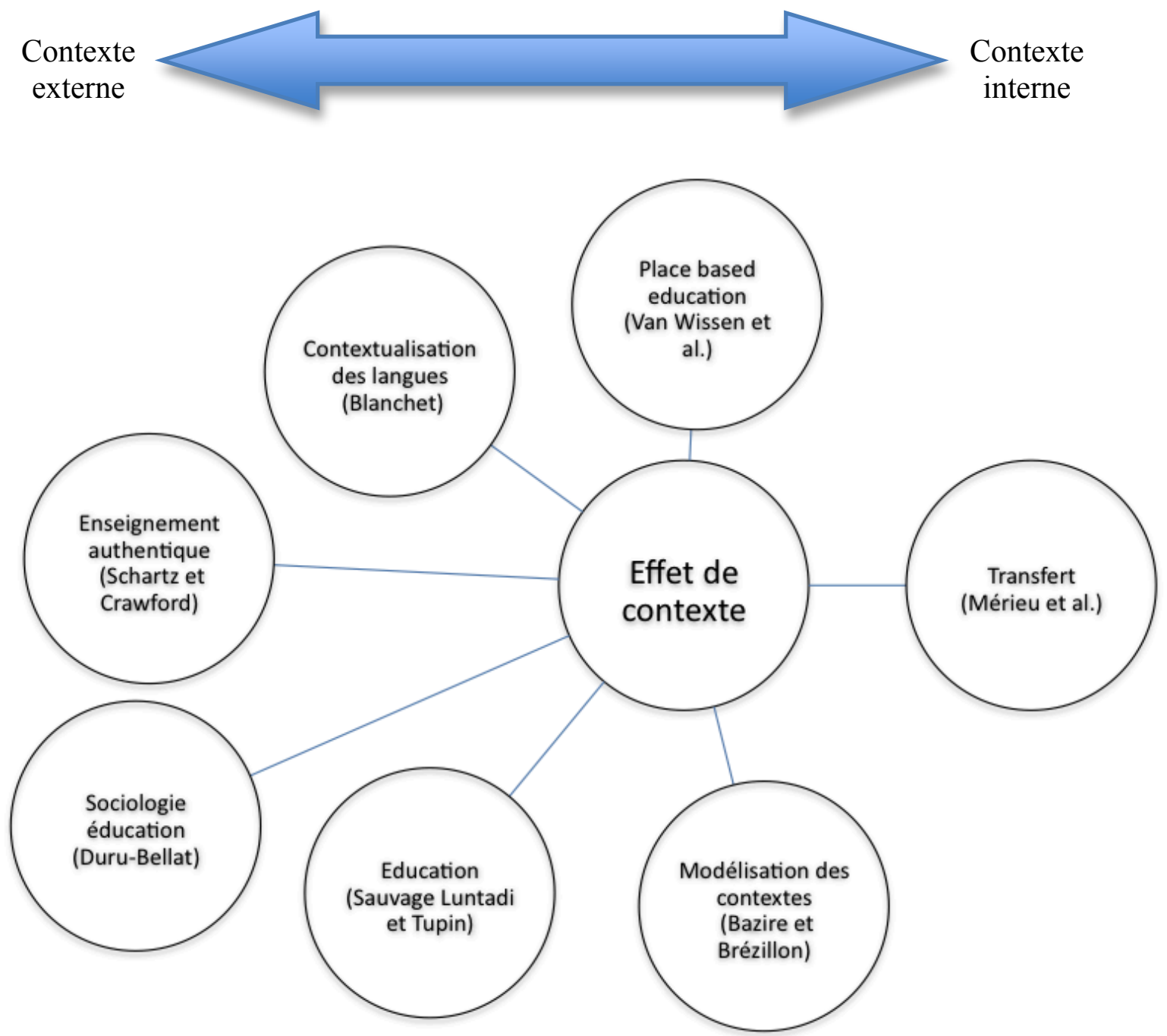

Figure 1 : Effet de contexte, un élément situé au niveau des contextes internes

Dans la partie droite de la Figure 1, d'autres travaux portant sur le transfert de connaissances (Mérieu, Develay, Durand et Mariani, 1996) s'intéressent à un contexte interne et proposent la contextualisation, la décontextualisation et la recontextualisation comme un processus cognitif oscillant entre des énoncés singuliers et généraux.

À l'instar d'autres recherches portant notamment sur la modélisation du contexte (Bazire et Brézillon, 2005 ; Van Eijck et Roth, 2010 ; Van Wissen, 2013), cet article vise à tisser des liens entre les contextes internes (potentiellement à l'origine d'effets de contexte) et les contextes externes. C'est dans cette perspective que le projet TEEC propose de modéliser l'émergence des effets de contexte à partir des écarts de contextes externes.

\subsection{Enseignements basés sur les effets de contextes et la modélisation du contexte}

Les enseignements basés sur les contextes présentent quatre caractéristiques selon Gilbert (2006) : un cadre social, spatial et temporel dans lequel les rencontres mentales avec les concepts sont situées; un environnement d'apprentissage de ces rencontres précisant la manière dont les tâches des acteurs et les événements sont construits et utilisés pour amener les notions; l'utilisation d'un langage spécifique lié à ces notions et une relation avec les 
connaissances extra-situationnelles. Les situations qui ont été produites dans le modèle présenté dans le cadre de cet article reposent spécifiquement sur l'idée de confronter des conceptions forgées dans différents contextes externes. Plus précisément, ces situations sont structurées selon un modèle d'apprentissage collaboratif à distance.

La modélisation des contextes externes est ici utilisée pour prédire l'émergence d'effets de contexte, événements qui sont observés et décrits selon plusieurs modalités d'analyse des interactions entre apprenants à partir d'enregistrements vidéo. Selon les itérations, la modélisation est synchrone ou antérieure à la réalisation du scénario pédagogique.

\section{Genèse du Projet TEEC et du modèle CLASH}

\subsection{Présentation des différents éléments (différences de contexte, instruments, calculateur, tableaux)}

Les premiers travaux qui devaient déboucher sur le projet TEEC se sont déroulés dans les locaux du CRREF en 2012, lors d'un séjour scientifique de Jacqueline Bourdeau. Les discussions sur les défis de l'enseignement en contexte nous ont conduits à élaborer un modèle pédagogique visant à mettre en lumière le rôle du contexte dans l'apprentissage des sciences, et à exploiter un contraste fort entre deux contextes, en prenant pour banc d'essai le Québec et la Guadeloupe. Il nous est ensuite apparu que pour concevoir un scénario pédagogique efficace, il nous fallait calculer le contraste entre deux contextes, ainsi que leurs paramètres respectifs. Le principe du calculateur de contexte MazCalc venait de naître. Si le modèle ainsi constitué, baptisé CLASH (Forissier et al. 2014), vise à mettre en jeu des contextes externes contrastés, le modèle d'apprentissage utilisé ne s'inscrit en aucune manière dans une compétition entre les élèves mais dans une logique de collaboration à distance (Dillenbourg, 1999) issue des approches Jigsaw (Aronson, 1997).

\subsection{Premières expérimentations}

Trois proto-expérimentations ont été menées en 2014 et 2015 : «Gounouy » en biologie, «Dlo » en sciences de l'eau et "Géothermie » en géologie. Gounouy signifie " grenouille » en créole guadeloupéen. En Guadeloupe, la plus petite grenouille du monde, l'hylode, est omniprésente dans les maisons et les jardins. Au Québec vit le ouaouaron, la plus grosse grenouille en Amérique du Nord. Deux enseignantes, Sophie Fécil en Guadeloupe et Julie Poulin au Québec, ont organisé les échanges entre les élèves de leur classe. Dlo signifie « eau » en créole guadeloupéen. Les élèves devaient étudier l'eau selon plusieurs paramètres, dans leur contexte respectif; ceux de Guadeloupe en bord de mer, et ceux du Québec sur les rives du fleuve Saint-Laurent. Dans le cadre du projet GEOTREF ${ }^{1}$, Claire Anjou a mené une expérimentation visant à faire collaborer des étudiants de master enseignement sur la Géothermie, opposant une géothermie haute énergie produisant de l'électricité en Guadeloupe (Usine de Bouillante) et des systèmes individuels de puits de chaleur servant au chauffage des habitations à Montréal.

\footnotetext{
${ }^{1}$ Plate-forme pluridisciplinaire d'innovation et de démonstration pour l'exploration et le développement de la GEOThermie haute énergie dans les REservoirs Fracturés, le projet GEOTREF est soutenu par l'Etat français au travers de financements d'Investissements d'Avenir.
} 


\section{Présentation du projet}

\subsection{Objectifs et hypothèses}

L'idée de départ du projet est de trouver comment intégrer le contexte dans l'apprentissage des sciences et comment étudier les modalités de cette intégration. Face à un enseignement trop souvent détaché du contexte, celui-ci pourrait être mis en lumière au bénéfice des élèves en provoquant un choc entre deux contextes contrastés, dans l'étude d'une même notion scientifique. Par exemple, deux groupes d'élèves, l'un en Guadeloupe et l'autre au Québec, conduisent une investigation sur la grenouille locale en cours de biologie, et parviennent à différencier ce qui est commun à l'espèce, et ce qui est dépendant du contexte, tropical ou laurentien.

Les différents objectifs du projet sont :

- élaborer une caractérisation des effets de contexte à partir d'indicateurs pour chaque domaine ;

- construire et tester un modèle d'enseignement basé sur l'émergence d'effets de contexte ;

- expérimenter le modèle et la stratégie mise en œuvre dans différents domaines et à plusieurs niveaux d'enseignement;

- élaborer un outil prédictif de l'émergence de ces effets de contexte, le tester et le rendre public ;

- mettre au point un modèle et des outils robustes pour concevoir des environnements d'apprentissage conscients du contexte.

En lien avec ces objectifs, trois hypothèses sont testées :

- un scénario pédagogique exploitant l'écart entre deux contextes externes suscite un changement conceptuel chez l'élève ;

- une modélisation du contexte permet de calculer les paramètres d'un contexte et l'écart entre deux contextes ;

- la prédiction des effets de contexte grâce à la modélisation sera validée par la mise en œuvre du scénario et l'évaluation des conceptions des élèves.

\subsection{Recherches basées sur le design et développement d'outils pédagogiques}

La méthodologie de recherche est de type Design-Based Research et comporte une série d'itérations permettant d'expérimenter le modèle et la stratégie avec des enseignants et des élèves, de valider les hypothèses et d'affiner les instruments. Les itérations planifiées pour 2017-2019 portent sur la géothermie, l'environnement, la sociohistoire et la littérature. Les analyses de données consistent en une analyse de traces et la détection des émotions durant les échanges. Deux instruments ont été conçus pour mettre en œuvre la stratégie pédagogique et de recherche. Le premier instrument est le scénario pédagogique qui structure les apprentissages et les enseignements par le biais d'activités similaires sur un thème commun et des échanges entre les élèves des deux groupes distants. Le deuxième instrument est un logiciel calculateur de contexte (MazCalc) qui permet de paramétrer les éléments de contexte de part et d'autre, de calculer l'écart entre les deux et donc de prédire les chances de succès d'un scénario. Un troisième instrument de développement est envisagé. Il s'agit d'un système tutoriel intelligent conscient du contexte, avec une modélisation du contexte qui permettra une adaptation du système à chaque nouveau contexte spécifique étudié. 


\subsection{Modélisation et identification des paramètres}

L'identification des paramètres est un enjeu majeur de la modélisation des contextes externes. Plusieurs difficultés ont été rencontrées au fil des itérations. En premier lieu, le nombre et les différents paramètres retenus dépendent des thèmes étudiés. Les thèmes scientifiques (Grenouille et Géothermie) semblent offrir des paramètres à la fois plus facilement identifiables et pour lesquels la littérature offre des valeurs de références disponibles. Les thèmes tels la sociohistoire et la littérature sont également paramétrables, mais la liste des paramètres, et surtout leurs valeurs, nécessitent un recours à plus de spécialistes que pour les autres thèmes. Cependant un certain nombre de paramètres, par exemple sur les contextes éducatifs, restent soumis à une intersubjectivité forte y compris à propos de thèmes scientifiques.

Au-delà du choix des paramètres retenus, des valeurs associées pour chaque contexte et des modalités de calcul des écarts de contexte pour chacun des paramètres, une attention particulière est portée sur les relations entre les différents paramètres, regroupés en "familles» de manière générique. Ces relations multiples peuvent être épistémologique, d'échelle, de thème ou de spécialité.

Dans toutes les itérations, l'implémentation des paramètres est faite en interrelation avec l'intention pédagogique. Le niveau et l'intention pédagogiques des enseignants sont pris en compte pour construire le modèle puis les résultats du modèle sont intégrés pour finaliser le scénario. Ce dernier est donc issu de collaborations entre tous les acteurs de la communauté de recherche impliquée (enseignants, didacticiens, experts et modélisateurs notamment).

\subsection{Analyses des données et production des résultats}

Les données recueillies lors des itérations sont soumises à trois analyses complémentaires :

- une analyse didactique basée sur les pré-tests et post-tests ainsi que sur des analyses de contenus des documents produits par les élèves et les enseignants ;

- une analyse des interactions entre les apprenants lors de phases de collaboration synchrone ;

- une analyse automatique des changements d'état émotionnel.

La comparaison de ces trois types de résultats vise à détecter et décrire les effets de contexte en prenant en compte les dimensions d'apprentissage, interactionnelle et émotive. Puis, un retour est effectué pour valider les prédictions émises par le modèle, dans le but d'affiner les hypothèses et de corriger ou d'améliorer les techniques et instruments mis en œuvre pour chaque itération.

\section{Coordination du projet, collaboration interdisciplinaire}

La mise en place des mécanismes de coordination pour faciliter la communication et le travail collaboratif des chercheurs en vue de produire les résultats escomptés par le projet, a pris en compte la diversité des personnes impliquées en termes de spécialité et de métier. 


\subsection{Les membres et équipes du projet}

Comme le montrent les Figures 2 et 3, le projet regroupe des membres issus de six institutions d'enseignement supérieur ou de recherche universitaire : l'université TÉLUQ, l'université du Québec à Montréal (UQAM), l'université des Antilles (UA), l'université Paris Descartes, le CNRS (Télécom ParisTech) et l'ESIA en France, répartis dans trois régions du monde distantes les unes des autres : les Antilles françaises (Pointe-à-Pitre), la France hexagonale (Paris) et le Québec (Montréal/Québec). Les professionnels impliqués sont des chercheurs et des enseignants. Ils se sont regroupés en équipes d'itérations et d'analyses de données. De plus, une équipe de pilotage du projet a été constituée. Elle est composée des responsables nationaux du projet ainsi que de la personne chargée de la coordination et du responsable de l'intégration des technologiques éducatives. Si besoin est, les responsables d'équipes sont invités à certaines réunions selon les avancées du projet.

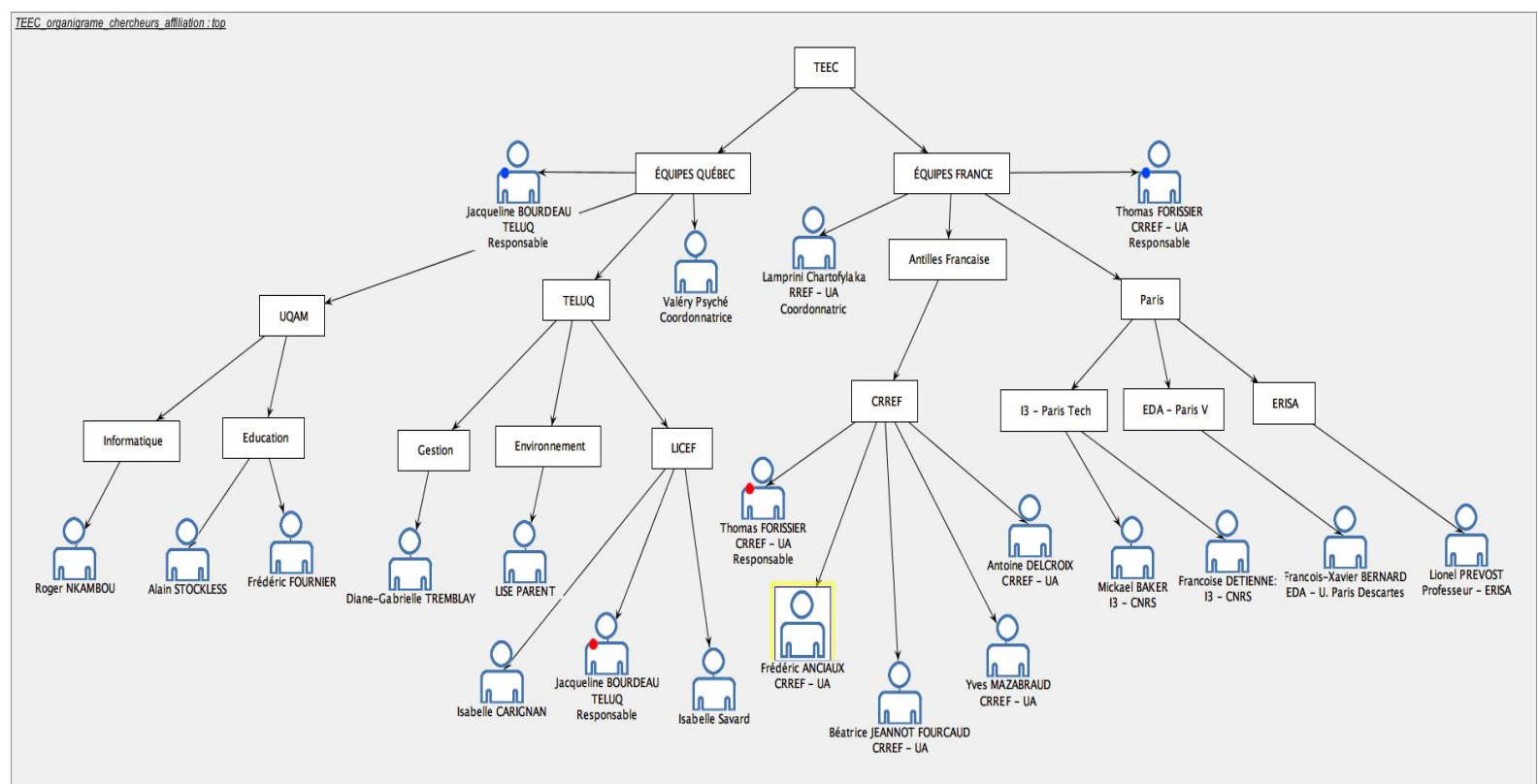

Figure 2 : Répartition des membres du projet TEEC par affiliation

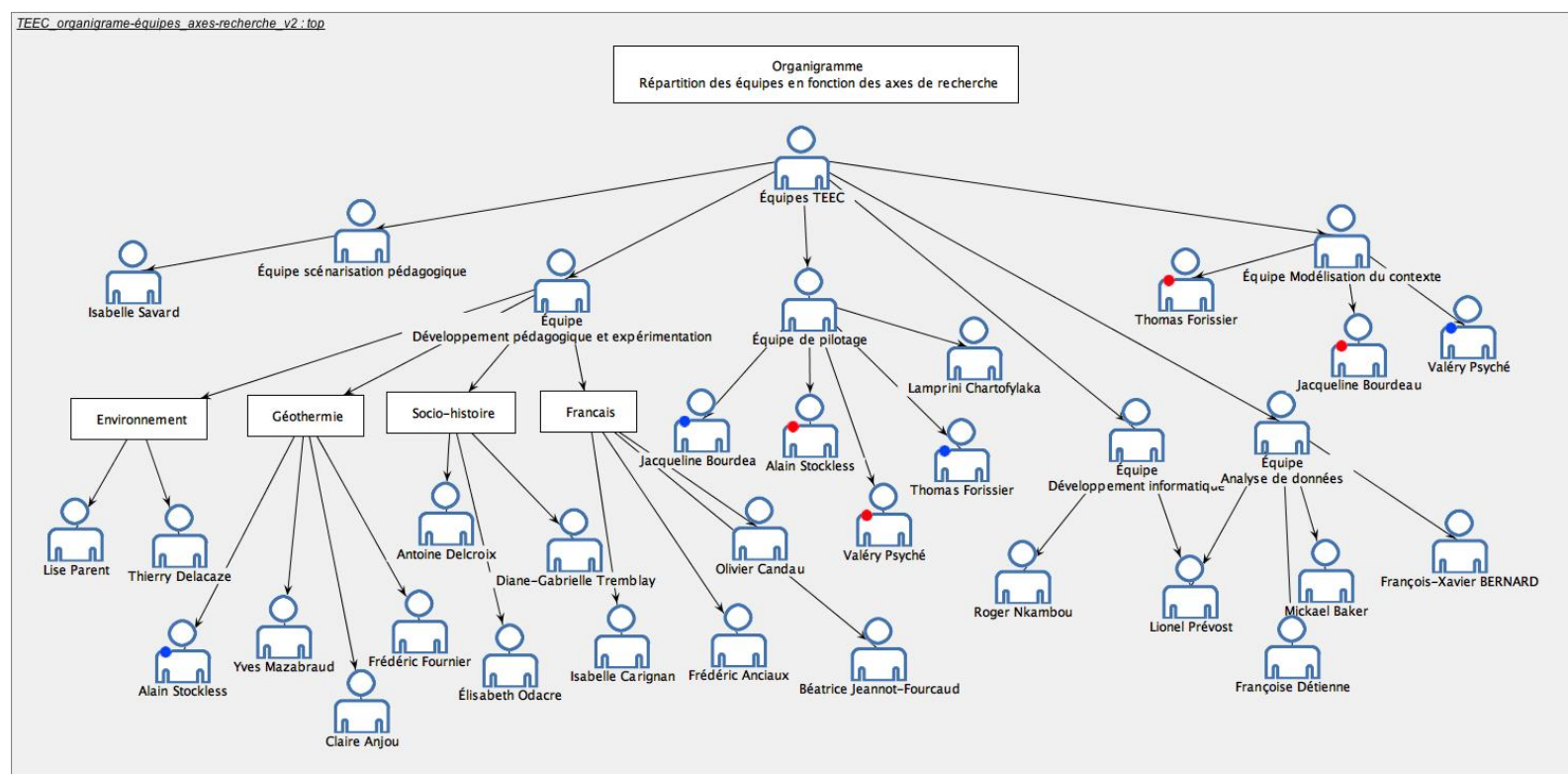

Figure 3 : Répartition des équipes en fonction des axes de recherche 


\subsection{Les activités principales du projet}

Les activités de l'équipe de pilotage consistent à superviser le projet et à accompagner les équipes de recherche. Elles se matérialisent par des rencontres hebdomadaires de suivi entre la coordinatrice et les responsables nationaux du projet. Le pilotage prévoit aussi l'organisation de réunions occasionnelles, en fonction des besoins et des avancées du projet, avec les responsables du développement pédagogique, du développement informatique et de l'analyse des données d'interactions issues des expérimentations. Trois rassemblements de la totalité des membres impliqués s'échelonnent également tout au long de la durée du projet.

Les activités de recherche (cf. Figure 4) sont réparties autour de la réalisation itérative des quatre expérimentations pédagogiques liées aux domaines d'études retenus (Géothermie, Littérature, Sociohistoire, Environnement), de la scénarisation pédagogique des expérimentations, de la modélisation du contexte didactique, du développement informatique du MazCalc et du CAITS, et de l'analyse de traces d'interactions entre les apprenants.

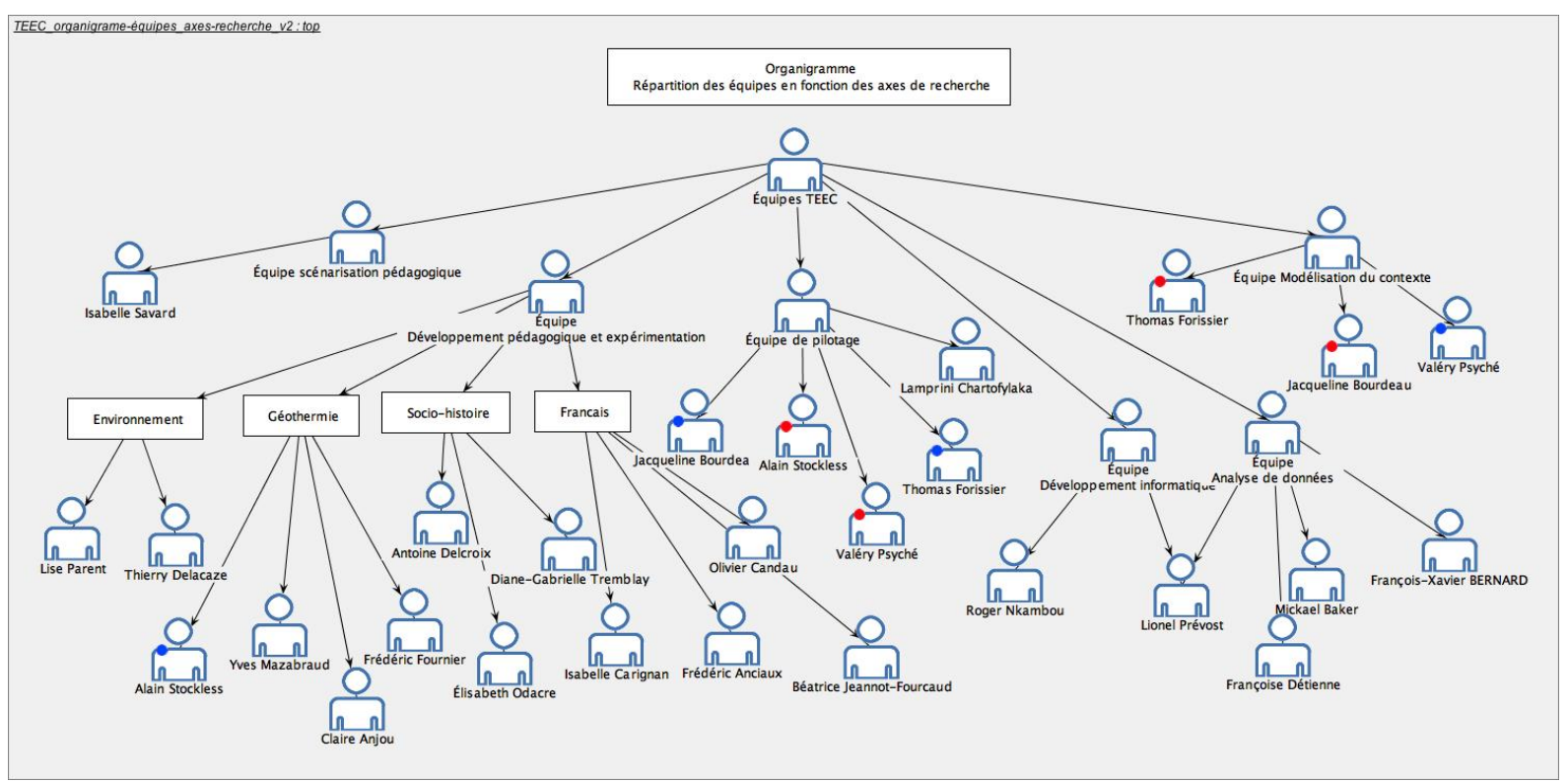

Figure 4 : Représentation des principales activités du projet TEEC

\subsection{Organisation}

Dans le cadre de la méthodologie DBR, une synchronisation des différentes expérimentations est importante. Le calendrier prévisionnel est présenté dans le Tableau 1. Le double objectif de construction de connaissances et de développement d'outils nécessite une attention particulière afin que les outils développés puissent être utilisés pour les différentes expérimentations lors des différentes itérations. Pour ce faire deux instruments de coordination ont été utilisés : une plateforme collaborative (Edmodo) et une plateforme de synchronisation de fichiers (Nextcloud). 
Expérimentation Sociohistoire

Planification des activités suivantes:

- Analyse de la tâche pédagogique : mars-avril 2017

- Arrimage des curricula québécois et français;

- Conception du scénario sociohistoire : juillet-août 2017

- Organisation de l'expérimentation : octobre-novembre 2017

\section{Thème Géothermie}

Planification des activités suivantes :

- Analyse de la tâche pédagogique : 2017

- Arrimage des curricula québécois et français ;

- Conception du scénario : février-mars 2017

- Organisation de l'expérimentation : septembre-octobre 2017

Thème Littérature

Planification des activités suivantes :

- Analyse de la tâche pédagogique : 2017

- Arrimage des curricula québécois et français ;

- Conception du scénario : septembre-décembre 2017;

- Organisation de l'expérimentation : janvier 2018 ;

\section{Thème Environnement}

Planification des activités suivantes :

- Analyse de la tâche pédagogique : 2017

- Arrimage des curricula québécois et français : ;

- Conception du scénario :

- Banane/pomme : 2018;

- $\quad$ Sucre/sirop d'érable : 2018

- Organisation de l'expérimentation :

- Banane/pomme : à déterminer ;

- Sucre/sirop d'érable : septembre 2018

Équipe Modèle de contexte - MazCalc

Volet métamodèle de contexte CLASH (février 2017 - 2018)

- Conception d'une ontologie du contexte. Équipe : C. Anjou, J. Bourdeau, V. Psyche, I. Savard

- Itération 1 du modèle : décembre 2017

- Itération 2 du modèle : 2018

Volet MazCalc (février 2017 - 2018)

- Implémentation du web service MazCalc, et des interfaces appropriées pour l'instanciation de différents contextes.

> Itération 1 du modèle : décembre 2017

Équipe Analyse de Données

Post-Doctorant (mars 2017-mars 2018)

- Analyse temps réel attentionnelle et émotionnelle des captations vidéo des interactions pédagogiques élèves-élèves et maître-élèves. Production d'indicateurs émotionnels (surprise, joie...) et/ou affectifs (proactif/réactif...).

Stage 1 (avril-juillet 2017)

- Intégration des indicateurs précédents dans le calculateur d'écart de contexte (MazCalc); première itération DBR

> Intégration des indicateurs dans MazCalc terminée : juillet 2017

Stage 2 (avril-juillet 2018)

- Intégration des indicateurs précédents dans le CAITS ; seconde itération DBR

> Intégration des indicateurs dans CAITS terminée : juillet 2018

Tableau 1 : Activités principales par équipe dans le cadre du projet TEEC 
Edmodo est une plateforme collaborative offrant des fonctionnalités de communication similaires à celles que l'on trouve sur certains réseaux sociaux (Facebook, par exemple) dans un environnement contrôlé. À cela s'ajoutent des fonctionnalités pédagogiques qui facilitent la gestion d'une ou plusieurs classes par un enseignant, mais aussi la gestion du travail collaboratif entre les enseignants et possiblement à l'intérieur d'une école. Il s'agit donc d'une application de réseautage social à l'intérieur d'un écosystème éducatif virtuel. Une des grandes difficultés du projet a été l'acceptation et l'appropriation de la plateforme collaborative Edmodo par l'ensemble des membres du projet TEEC. Il convient de décrire les activités de collaborations avec la plateforme Edmodo, puis d'analyser ce processus. Les principaux utilisateurs d'Edmodo sont l'enseignant, l'élève et le parent, mais on peut prévoir d'autres types d'utilisateurs hors de la classe. Nous avons de ce fait pu répondre aux besoins de travail collaboratif des membres du projet TEEC qui étaient essentiellement les mêmes que ceux d'un enseignant avec ses collègues enseignants : (1) envoyer des messages à un groupe et les afficher au «mur» du groupe ; (2) partager des documents, des notes, des liens ; (3) structurer et stocker en ligne ces documents ; (4) créer des documents de la suite Microsoft en mode collaboratif; (5) planifier des événements ; (6) créer des sondages ; (7) visualiser les fils de discussion, témoins des interactions entre les membres ; (8) insérer et afficher sur le mur du groupe des ressources multimédia.

Bien que la structuration et la gestion des données arrivent dans un troisième temps, la réflexion a été amorcée dès le lancement du projet. Quelques éléments essentiels pour les chercheurs et dont il fallait tenir compte portaient sur le stockage des données produites lors des expérimentations dans un environnement sécurisé à la TELUQ, et sur la nécessité de préserver la propriété intellectuelle des chercheurs et la confidentialité de ces données. Ceci a été pris en compte dans la suite du projet et a mené à l'exploration d'un réseau privé virtuel (VPN), puis à son abandon en faveur de la plateforme Nextcloud. Il s'est avéré que Nextcloud répondait mieux aux besoins des chercheurs dans la mesure où l'objectif principal de Nextcloud est de donner aux utilisateurs la pleine souveraineté sur leurs données. Parmi les nombreuses fonctionnalités disponibles, cette plateforme permet de stocker, structurer, partager et synchroniser des fichiers de données entre plusieurs utilisateurs de façon sécuritaire et confidentielle.

\section{Discussion}

\subsection{Difficultés rencontrées}

Nous avons rencontré plusieurs difficultés dans la mise en œuvre de cette recherche :

- Résistances au changement technologique et dysfonctionnement dans la communication: tout d'abord, il y a eu une résistance de certains chercheurs à l'utilisation de la plateforme de communication choisie, Edmodo. Ceci a occasionné un dysfonctionnement dans la communication puisque cela nous a contraint à envoyer des messages via la plateforme mais également par d'autres moyens.

- Limites fonctionnelles de la plateforme Edmodo: cette plateforme est encore en version Beta et n'est pas encore stable. Ainsi, nous sommes limités à différents niveaux en ce qui concerne les potentialités de la plateforme, et les fonctionnalités évoluent sans qu'on n'en soit toujours informé. Parmi les contraintes les plus importantes, on note que le lanceur d'application est limité. Il est donc impossible de lancer la plupart des applications désirées à partir de Edmodo.

- Difficultés de trouver des enseignants : la faisabilité du projet repose en grande partie sur l'implication bénévole d'enseignants et de leur classe dans chaque thème 
(Géothermie, Littérature, etc.). Certains thèmes ont eu des difficultés à démarrer faute de trouver des enseignants (et leur classe) qui désiraient participer au projet de recherche.

- Difficultés liées au manque de concordance entre certains curricula d'enseignement en Guadeloupe et au Québec: la différence la plus importante a été relevée pour l'enseignement supérieur. Il est parfois nécessaire de travailler sur la convergence des notions présentes dans les curricula.

\subsection{Enjeux}

Le premier enjeu est celui de mettre à disposition de la communauté un modèle original et validé d'exploitation du contexte dans les apprentissages, d'un service Web de calcul des écarts de contexte ainsi que d'un modèle de système intelligent conscient du contexte. Les résultats permettent de qualifier l'efficacité du scénario et des instruments développés.

S'inscrivant pleinement dans la stratégie qui vise à faire entrer l'école dans l'ère du numérique en France, la production d'une innovation clés en main, reposant sur un modèle validé, instrumenté et dont les conditions d'adoption sont également documentées, constitue la principale retombée sociétale de ce projet. Cette innovation possède un caractère générique, pouvant en effet être déployée dans de nombreuses situations, plusieurs disciplines et niveaux scolaires. L'enjeu réside dans l'évolution des pratiques éducatives vers des approches centrées sur les territoires et les contextes des élèves, mais ouvertes sur le monde grâce au numérique et aux collaborations citoyennes internationales.

Trois questions scientifiques nouvelles concernant les relations entre didactiques et contextes sont apparues au cours de ce projet. La première concerne les modalités de simplification des enseignements basés sur les effets de contexte afin que cette pratique puisse réellement intégrer l'espace scolaire. La deuxième est axée sur la généralisation, la transposition à d'autres domaines d'apprentissages que ceux liés aux sciences. Enfin, la troisième interroge l'échelle spatiale pertinente pour opposer des contextes.

\section{Remerciements}

Les auteurs souhaitent remercier l'ensemble des acteurs qui ont permis la réalisation du projet TEEC et en premier lieu l'Agence Nationale de la Recherche et le Fond de Recherche Société et Culture du Québec.

\section{Références bibliographiques}

Aronson, E. (1997). The jigsaw classroom: Building cooperation in the classroom. Scott Foresman et Company.

Bazire, M. et Brézillon, P. (2005). Understanding context before to use it. LNAI 3554, 29-40. doi:10.1007/11508373 3.

Blanchet, P. (2009). "Contextualisation didactique », de quoi parle-t-on? Le français à l'université. Repéré à http://eprints.aidenligne-francais-universite.auf.org/147/1/pdf.pdf.

De Lacaze, T. (2015). Contextualisation de l'éducation au développement durable en Guadeloupe : conceptions d'acteurs socioéconomiques, d'élèves du cycle 3 et pratiques enseignantes. Thèse de doctorat, université des Antilles. 
Delcroix, A. Forissier, T. et Anciaux, F. (2013). Vers un cadre d'analyse opérationnel des phénomènes de contextualisation didactique. Dans F. Anciaux, T. Forissier et L. F. Prudent (dir.), Contextualisations didactiques. Approches théoriques (p. 141-185). Paris : L'Harmattan.

Dillenbourg, P. (1999). Collaborative learning: Cognitive and computational approaches. advances in learning and instruction series. New York : Elsevier.

Duru-Bellat, M. (2002). Les inégalités sociales à l'école: Genèse et mythes. Paris : Presses Universitaires de France. doi:10.3917/puf.duru.2002.01.

Flanagan J. C. (1954). The critical incident technique. Psychological Bulletin. 51(4).

Forissier, T., Bourdeau, J. Mazabraud, Y. et Nkambou R, (2014) Computing the Context Effect for Science Learning. Dans P. Brézillon et A. J. Gonzalez (dir.), Context in Computing (p. 255-269). New York : Springer.

Gilbert, J. (2006). On the nature of Context in chemical education. International Journal of Science Education, 28(9), 957-976. doi:10.1080/09500690600702470.

King, D. et Ritchie, S. M. (2012). Second international handbook of science education, 69-79.

Meirieu, P., Develay, M., Durand, C. et Mariani, Y. (1996). Le transfert de connaissances en formation initiale et en formation continue. Lyon : CRDP.

Merlo-Leurette, S. et Forissier, T. (2009). La contextualisation dans l'enseignement des sciences et techniques en Guadeloupe. Grand N, 83, 19-27.

Odacre, E. et Delcroix. A. (2014). National diploma vs local employability : A french paradox at work in Guadeloupe. 19th General Meeting and Biennial Conference "Harnessing Science and Technology to create knowledge-base Economies and preserve Caribbean Ecosystems", 2014, Trinidad and Tobago.

Podschuweit, S. et Bernholt, S. (2017). Composition-Effects of Context-based Learning Opportunities on Students' Understanding of Energy. Research in Science Education, 136.

Schwartz, R.S. et Crawford, B.A. (2006). Authentic Scientific Inquiry As Context For Teaching Nature Of Science: Identifying Critical Element. Dans L.B. Flick et N. G. Lederman N.G. (dir.). Scientific Inquiry and Nature of Science. Science \& Technology Education Library, vol 25. Dordrecht : Springer.

Sauvage Luntadi, L. et Tupin, F. (2012). La compétence de contextualisation au coeur de la situation d'enseignement-apprentissage. Phronesis, 1(1), 102-117. doi:10.7202/1006488ar.

Van Eijck, M. et Roth, W.M. (2010). Towards a chronotopic theory of "place" in place-based education. Cult. Stud. Sci. Educ. 5(4), 869-898. doi:10.1007/s11422-010-9278-2

VanWissen, A., Kamphorst, B. et Van Eijk, R. (2013). A constraint-based approach to context. Dans P. Brésillon et R. Dapoigny (dir.), Modeling and Using Context. CONTEXT 2013. Lecture Notes in Computer Science, vol. 8175. Berlin, Heidelberg : Springer

Weinburgh, M. (1995). Gender differences in student attitudes toward science: a metaanalysis of the literature from 1970 to 1991 Journal of Research in Science Teaching, 32, 387-398. 